Article

\title{
Conformal Symmetry and Supersymmetry in Rindler Space
}

\author{
Jan-Willem van Holten ${ }^{1,2}$ (D) \\ 1 Nikhef, 1098XG Amsterdam, The Netherlands; v.holten@nikhef.nl \\ 2 Lorentz Institute, Leiden University, Niels Bohrweg 2, 2333CA Leiden, The Netherlands
}

Received: 27 July 2020; Accepted: 31 August 2020; Published: 4 September 2020

\begin{abstract}
This paper addresses the fate of extended space-time symmetries, in particular conformal symmetry and supersymmetry, in two-dimensional Rindler space-time appropriate to a uniformly accelerated non-inertial frame in flat 1+1-dimensional space-time. Generically, in addition to a conformal co-ordinate transformation, the transformation of fields from Minkowski to Rindler space is accompanied by local conformal and Lorentz transformations of the components, which also affect the Bogoliubov transformations between the associated Fock spaces. I construct these transformations for massless scalars and spinors, as well as for the ghost and super-ghost fields necessary in theories with local conformal and supersymmetries, as arising from coupling to two-dimensional (2-D) gravity or supergravity. Cancellation of the anomalies in Minkowski and in Rindler space requires theories with the well-known critical spectrum of particles that arise in string theory in the limit of infinite strings, and it is relevant for the equivalence of Minkowski and Rindler frame theories.
\end{abstract}

Keywords: Rindler space; conformal symmetry; supersymmetry; Bogoliubov transformations

\section{Introduction}

Conformal field theories have become an important tool in our theories of nature, both in the context of particle physics and quantum gravity and in the context of condensed matter [1-3]. Conformal field theories in two dimensions are especially relevant in string theory [4], the $\mathrm{AdS}_{3}$-CFT correspondence and condensed matter [5,6], and as a model for quantum field theories also in higher dimensions.

Effectively conformal field theories in two space-time dimensions can be formulated as theories of massless fields in a Minkowski background. This is the point of view used for practical purposes in the present paper. In fact, our focus is on the description of these theories in a non-standard frame: that of a uniformly accelerated observer [7]. The interest in this frame stems from the non-inertial effects that manifest themselves in the presence of a horizon and finite-temperature behaviour: the Unruh effect [8-11], a counterpart of the Hawking effect in black-hole space-times [12,13] and in cosmology [14]. More generally, it has been found that free field theories in Rindler space describe the near-horizon behaviour of quantum field theories in the presence of black holes, which is particularly relevant in the context of AdS-CFT correspondence [15]. Extensive reviews of field theories in Rindler space can be found in refs. [16,17].

In two space-time dimensions, local conformal transformations can be used to cast the line element in the form

$$
d s^{2}=\rho^{2}(x) \eta_{\mu v} d x^{\mu} d x^{\nu},
$$

where $\eta_{\mu v}$ is the flat Minkowski metric and $\rho^{2}(x)$ is the conformal factor, which is constrained by the topology. In the present paper, we restrict ourselves to inertial observers in infinite flat Minkowski 
space for which $\rho^{2}(x)=1$, and to accelerated observers in the associated Rindler space, a subset of Minkowski space consisting of two branches: the right and left Rindler wedges $R$ and $L$, defined by

$$
\begin{aligned}
& R: \quad x>0 \text { and } \quad-x<t<x ; \\
& L: \quad x<0 \text { and } \quad x<t<-x .
\end{aligned}
$$

Each wedge is parametrized by a conformally flat metric in terms of co-ordinates $(\tau, \xi)$ that are associated with an accelerated observer:

$$
\begin{aligned}
& R: \quad x=\frac{1}{a} e^{a \xi} \cosh a \tau, \quad t=\frac{1}{a} e^{a \xi} \sinh a \tau, \\
& L: \quad x=-\frac{1}{a} e^{-a \xi} \cosh a \tau, \quad t=\frac{1}{a} e^{-a \xi} \sinh a \tau .
\end{aligned}
$$

In this parametrization, the line element is of the form (1) with conformal factor $\rho=e^{ \pm a a^{\tau}}$ :

$$
\begin{aligned}
& R: \quad d s^{2}=e^{2 a \xi}\left(-d \tau^{2}+d \xi^{2}\right), \\
& L: \quad d s^{2}=e^{-2 a \xi}\left(-d \tau^{2}+d \xi^{2}\right) .
\end{aligned}
$$

The parameter $a$ in the co-ordinate transformation represents the proper accelaration in the Minkowski frame of a body with Rindler co-ordinate $\xi=0$ at the moment it crosses the $x$-axis. It can be absorbed in a further rescaling of the Rindler co-ordinates $(\tau, \xi)$ by $1 / a$ and a shift of $\xi$ by $\xi_{0}=\ln a$; as a result the line element reduces to (4) with $a=1$. This is our standard choice in the following. In all of this paper, units are chosen, such that $c=\hbar=1$.

\section{Massless Fields in 2-D Minkowski Space}

To fix conventions and for later reference, in this section we briefly review free field theories of massless scalar fields (bosons) and massless spinor fields (fermions) in two-dimensional (2-D) Minkowski space. The theory of a real, free scalar field $\varphi(x)$ is defined by the action

$$
S_{b}=\frac{1}{2} \int_{M} d^{2} x\left[\left(\partial_{t} \varphi\right)^{2}-\left(\partial_{x} \varphi\right)^{2}\right] .
$$

The solutions of the associated Klein-Gordon equation

$$
\left(\partial_{t}^{2}-\partial_{x}^{2}\right) \varphi=0,
$$

are superpositions of left- and right-moving fields

$$
\varphi(t, x)=\varphi_{+}(t+x)+\varphi_{-}(t-x) .
$$

For definiteness in the following we concentrate on left-moving fields $\varphi_{+}$. Its conjugate field momentum is $\pi_{+}=\partial_{t} \varphi_{+}$, and the time-evolution of all functionals of the field is governed by the hamiltonian

$$
H_{b}=\frac{1}{2} \int_{-\infty}^{\infty} d x\left[\pi_{+}^{2}+\left(\partial_{x} \varphi\right)^{2}\right],
$$

using the equal-time commutation relation

$$
\left[\varphi_{+}(t, x), \pi_{+}(t, y)\right]=i \delta(x-y) .
$$


In a plane-wave basis, the field is expanded as

$$
\varphi_{+}(t+x)=\int_{0}^{\infty} \frac{d k}{2 \pi \sqrt{2 k}}\left(a_{k} e^{-i k(t+x)}+a_{k}^{*} e^{i k(t+x)}\right),
$$

the domain of momenta $k$ for left-moving fields being $[0, \infty)$. The plane-wave mode operators are defined inversely by

$$
a_{k}=\frac{i}{\sqrt{2 k}} \int_{-\infty}^{\infty} d x e^{i k(t+x)} \stackrel{\leftrightarrow}{\partial} t \varphi_{+}, \quad a_{k}^{*}=-\frac{i}{\sqrt{2 k}} \int_{-\infty}^{\infty} d x e^{-i k(t+x)} \stackrel{\leftrightarrow}{\partial} t \varphi_{+} .
$$

The commutation relation (9) is equivalent to

$$
\left[a_{k}, a_{q}^{*}\right]=2 \pi \delta(k-q)
$$

and the hamiltonian after normal-ordering is

$$
: H_{b}:=\int_{0}^{\infty} \frac{d k}{2 \pi} k a_{k}^{*} a_{k} .
$$

Finally the ground state of the field is the bosonic Minkowski vacuum $|0\rangle_{b}$ :

$$
\forall k: \quad a_{k}|0\rangle_{b}=0 .
$$

Next, we discuss spinor fields, using the conventions collected in Appendix A. A general Dirac spinor is a complex two-component object; however, 2-D Minkowski space-time allows Majorana-Weyl spinors that are real single-component objects representing left- or right-moving solutions of the massless Dirac equation. Indeed, in the Majorana representation making use of the charge conjugation operator $C$ the Dirac action takes the diagonal form

$$
S_{f}=\frac{i}{2} \int_{M} d^{2} x \lambda^{T} C \not \partial \lambda, \quad C \not \partial=\left(\begin{array}{cc}
\partial_{t}-\partial_{x} & 0 \\
0 & \partial_{t}+\partial_{x}
\end{array}\right) .
$$

and for a 2-component Majorana spinor

$$
\lambda=C \bar{\lambda}^{T}=\left[\begin{array}{l}
\lambda_{+} \\
\lambda_{-}
\end{array}\right],
$$

the associated Dirac equation splits into independent equations

$$
\left(\partial_{t}-\partial_{x}\right) \lambda_{+}=0, \quad\left(\partial_{t}+\partial_{x}\right) \lambda_{-}=0,
$$

representing separate left- and right moving fields. Again, for definiteness, we concentrate on left-moving fields $\lambda_{+}(t+x)$. The time-evolution of functionals of this field is governed by the hamiltonian

$$
H_{f}=\frac{i}{2} \int_{-\infty}^{\infty} d x \lambda_{+} \partial_{x} \lambda_{+}
$$

and the equal-time anticommutation relation

$$
\left\{\lambda_{+}(t, x), \lambda_{+}(t, y)\right\}=\delta(x-y) .
$$

Defining the plane-wave expansion by

$$
\lambda_{+}(t+x)=\int_{0}^{\infty} \frac{d k}{2 \pi}\left(\alpha_{k} e^{-i k(t+x)}+\alpha_{k}^{*} e^{i k(t+x)}\right),
$$


with inverse

$$
\alpha_{k}=\frac{i}{2 k} \int_{-\infty}^{\infty} d x e^{i k(t+x)} \stackrel{\leftrightarrow}{\partial} t \lambda_{+}, \quad \alpha_{k}^{*}=-\frac{i}{2 k} \int_{-\infty}^{\infty} d x e^{-i k(t+x)} \overleftrightarrow{\leftrightarrow}_{t} \lambda_{+}
$$

the anti-commutation relations translate to

$$
\left\{\alpha_{k}, \alpha_{q}^{*}\right\}=2 \pi \delta(k-q)
$$

After normal-ordering, the hamiltonian (17) becomes

$$
: H_{f}:=\int_{0}^{\infty} \frac{d k}{2 \pi} k \alpha_{k}^{*} \alpha_{k}
$$

with ground state the fermionic Minkowski vacuum $|0\rangle_{f}$ :

$$
\forall k: \quad \alpha_{k}|0\rangle_{f}=0 .
$$

The theory of a combined massless scalar and spinor possesses supersymmetry. The combined hamiltonian

$$
H=: H_{b}:+: H_{f}:=\int_{0}^{\infty} \frac{d k}{2 \pi} k\left(a_{k}^{*} a_{k}+\alpha_{k}^{*} \alpha_{k}\right),
$$

allows a square root

$$
Q=\int_{0}^{\infty} \frac{d k}{2 \pi} \sqrt{k}\left(a_{k}^{*} \alpha_{k}+\alpha_{k}^{*} a_{k}\right),
$$

such that $Q^{2}=H$ and $[Q, H]=0$. The supersymmetry transformations of the mode operators have the standard form

$$
\begin{aligned}
& {\left[a_{k}, Q\right]=\sqrt{k} \alpha_{k}, \quad\left[a_{k}^{*}, Q\right]=-\sqrt{k} \alpha_{k}^{*},} \\
& \left\{\alpha_{k}, Q\right\}=\sqrt{k} a_{k}, \quad\left\{\alpha_{k}^{*}, Q\right\}=\sqrt{k} a_{k}^{*} .
\end{aligned}
$$

The common Minkowski vacuum $|0\rangle_{M}=|0\rangle_{b} \otimes|0\rangle_{f}$ is supersymmetric:

$$
Q|0\rangle_{M}=0 .
$$

It is straightforward to generalize the contructions to theories of a larger number of bosons and fermions. With equal numbers of $N$ bosons and $N$ fermions there are, in general, $N$ ! supersymmetries, associating every boson with each of the fermions, as at this point the various boson and fermion fields are indistinguishable and related by an $O(N) \otimes O(N)$ symmetry.

\section{Massless Fields in 2-D Rindler Space}

Two-dimensional Rindler space is the space-time described by the metric (1), with the conformal factor specified for the left and right wedges by (4). It is therefore obvious that in Rindler space

$$
\sqrt{-g} g^{\mu \nu}=\eta^{\mu \nu}
$$

Moreover the scalar field in the Rindler frame is related to the original Minkowski field by the co-ordinate transformation (3): $\varphi_{R}(\tau, \xi)=\varphi[t(\tau, \xi), x(\tau, \xi)]$. It follows that the action for scalar fields in Rindler space is formally identical to that in Minkowski space:

$$
S_{R b}=\frac{1}{2} \int_{R} d \tau d \xi\left[\left(\partial_{\tau} \varphi_{R}\right)^{2}-\left(\partial_{\xi} \varphi_{R}\right)^{2}\right] .
$$

which holds both in the right and left wedge of Rindler space, independent of the different conformal factors $\rho=e^{ \pm a \zeta}$. Concentrating again on the left-moving solutions of the associated Klein-Gordon 
equation and following the same procedures, as described for bosons in Section 2, we can represent them in terms of the plane-wave expansion

$$
\varphi_{R+}(\tau+\xi)=\int_{0}^{\infty} \frac{d \kappa}{2 \pi \sqrt{2 \kappa}}\left(b_{\kappa} e^{-i \kappa(\tau+\xi)}+b_{\kappa}^{*} e^{i \kappa(\tau+\xi)}\right),
$$

where

$$
b_{\kappa}=\frac{i}{\sqrt{2 \kappa}} \int_{-\infty}^{\infty} d \xi e^{i \kappa(\tau+\xi)} \stackrel{\leftrightarrow}{\partial} \tau \varphi_{R+}, \quad b_{\kappa}^{*}=-\frac{i}{\sqrt{2 \kappa}} \int_{-\infty}^{\infty} d \xi e^{-i \kappa(\tau+\xi)} \stackrel{\leftrightarrow}{\partial} \tau \varphi_{R+} .
$$

Free-field dynamics is generated by introducing the hamiltonian

$$
H_{R b}=\int_{0}^{\infty} \frac{d \kappa}{4 \pi} \kappa\left(b_{\kappa}^{*} b_{\kappa}+b_{\kappa} b_{\kappa}^{*}\right)
$$

together with the commutation relation

$$
\left[b_{\kappa}, b_{\sigma}^{*}\right]=2 \pi \delta(\kappa-\sigma)
$$

Standard procedure would replace $H_{R b}$ by its normal-ordered form, but normal ordering is now ambiguous as the result is different in terms of the mode operators $\left(b_{\kappa}, b_{\kappa}^{*}\right)$ or in terms the original mode operators $\left(a_{k}, a_{k}^{*}\right)$. Indeed, the Rindler vacuum $|0\rangle_{R b}$ defined by

$$
b_{\kappa}|0\rangle_{R b}=0,
$$

is not the same as the bosonic vacuum $|0\rangle_{b}$ in the original Minkowski space, as discussed below.

Translating the free fermion theory to Rindler space is somewhat more involved. Starting from the Dirac-Majorana action on a general space-time manifold $\mathcal{M}$

$$
S_{\mathcal{M} f}=\frac{i}{2} \int_{\mathcal{M}} d^{2} x \sqrt{-g} \lambda^{T} C \not D \lambda, \quad \not D=\gamma^{a} e_{a}^{\mu}\left(\partial_{\mu}-\frac{1}{2} \omega_{\mu}^{a b} \sigma_{a b}\right),
$$

where $e_{a}^{\mu}$ is the inverse 2-bein, $\omega_{\mu}^{a b}$ the associated spin connection and $\sigma_{a b}$ the generators of 2-D Lorentz transformations in tangent spinor space; as explained in Appendix A, we find that in a conformally flat space-time (1) the Dirac operator in the Majorana representation takes the form

$$
\sqrt{-g} C \not D=\sqrt{\rho}\left(\begin{array}{cc}
\partial_{\tau}-\partial_{\xi} & 0 \\
0 & \partial_{\tau}+\partial_{\tilde{\zeta}}
\end{array}\right) \sqrt{\rho}
$$

In addition, the transformation from Minkowski to Rindler co-ordinates also requires a $\tau$-dependent Lorentz transformation of the spinor fields; this is also explained in Appendix A. The upshot is that, upon redefining the spinor fields in the $R$ - and $L$-wedge of Rindler space by

$$
\begin{array}{ll}
R: & \psi_{ \pm}(\tau, \xi)=e^{ \pm a(\tau \pm \xi) / 2} \lambda_{ \pm}[t(\tau, \xi), x(\tau, \xi)], \\
L: & \psi_{ \pm}(\tau, \xi)=e^{\mp a(\tau \pm \xi) / 2} \lambda_{ \pm}[t(\tau, \xi), x(\tau, \xi)],
\end{array}
$$

the Dirac-Majorana action in a conformally flat space-time, like Rindler space, reduces to

$$
S_{R f}=\frac{i}{2} \int_{R} d \tau d \xi\left[\psi_{+}\left(\partial_{\tau}-\partial_{\tilde{\xi}}\right) \psi_{+}+\psi_{-}\left(\partial_{\tau}+\partial_{\xi}\right) \psi_{-}\right]
$$


The spinor fields $\psi_{ \pm}$have the same formal properties in Rindler space as the spinor fields $\lambda_{ \pm}$in Minkowski space; concentrating on the left-moving field, we introduce the plane-wave expansion

$$
\psi_{+}(\tau+\xi)=\int_{0}^{\infty} \frac{d \kappa}{2 \pi}\left(\beta_{\kappa} e^{-i \kappa(\tau+\xi)}+\beta_{\kappa}^{*} e^{i \kappa(\tau+\xi)}\right),
$$

where

$$
\beta_{\kappa}=\frac{i}{2 \kappa} \int_{-\infty}^{\infty} d x e^{i \kappa(\tau+\xi)} \overleftrightarrow{\partial}_{\tau} \psi_{+}, \quad \beta_{\kappa}^{*}=-\frac{i}{2 \kappa} \int_{-\infty}^{\infty} d x e^{-i \kappa(\tau+\xi)} \stackrel{\leftrightarrow}{\partial}_{\tau} \psi_{+} .
$$

In terms of mode operators the dynamics is defined by the hamiltonian

$$
H_{R f}=\int_{0}^{\infty} \frac{d \kappa}{4 \pi} \kappa\left(\beta_{\kappa}^{*} \beta_{\kappa}-\beta_{\kappa} \beta_{\kappa}^{*}\right),
$$

and the anti-commutator

$$
\left\{\beta_{\kappa}, \beta_{\sigma}^{*}\right\}=2 \pi \delta(\kappa-\sigma) .
$$

Again, in the pure-fermion theory we renounce normal ordering in view of its ambiguity. However, in the special case of a supersymmetric theory with equal numbers of bosons and fermions the normal-ordering contributions of bosons and fermions cancel, and therefore normal ordering becomes unambiguous. In that case, we can take the full hamiltonian to be

$$
H_{R}=H_{R b}+H_{R f}=\int_{0}^{\infty} \frac{d \kappa}{2 \pi} \kappa\left(b_{\kappa}^{*} b_{\kappa}+\beta_{\kappa}^{*} \beta_{\kappa}\right) .
$$

Clearly it is unique, owing to the existence of the supercharge

$$
Q_{R}=\int_{0}^{\infty} \frac{d \kappa}{2 \pi} \sqrt{\kappa}\left(b_{\kappa}^{*} \beta_{\kappa}+\beta_{\kappa}^{*} b_{\kappa}\right),
$$

with the algebraic properties

$$
Q_{R}^{2}=H_{R}, \quad\left[Q_{R}, H_{R}\right]=0 .
$$

\section{Relating Rindler and Minkowski Fields}

In the Rindler wedges, the field theories of Sections 2 and 3 are related by the co-ordinate transformation (3); they describe the same physics in different frames: with respect to an inertial observer using the Minkowski metric, or with respect to an accelerated observer using the conformally flat metrics (4). Therefore, also the Hilbert spaces and the observables in the two descriptions are related; the relation actually is provided by a Bogoliubov transformation between the mode operators $[8,13,16-19]$.

For the scalar fields, the relation is established starting from (30) and inserting for $\varphi_{R+}$ its Minkowski space expression, Equation (10). Choosing units to fix $a=1$ in the following and introducing the Minkowski light-cone co-ordinate

$$
z=t+x=e^{\tau+\xi},
$$

the Rindler mode operators in the $R$-wedge are expressed in terms of the Minkowski ones by

$$
\begin{aligned}
& b_{R \kappa}=\frac{1}{2 \pi \sqrt{2 \kappa}} \int_{0}^{\infty} \frac{d k}{\sqrt{2 k}} \int_{0}^{\infty} \frac{d z}{z} z^{i \kappa}\left[(\kappa+k z) a_{k} e^{-i k z}+(\kappa-k z) a_{k}^{*} e^{i k z}\right], \\
& b_{R \kappa}^{*}=-\frac{1}{2 \pi \sqrt{2 \kappa}} \int_{0}^{\infty} \frac{d k}{\sqrt{2 k}} \int_{0}^{\infty} \frac{d z}{z} z^{-i \kappa}\left[(\kappa-k z) a_{k} e^{-i k z}+(\kappa+k z) a_{k}^{*} e^{i k z}\right] .
\end{aligned}
$$


By appropriate choice of contour, the integrals over $z$ can be performed in the complex plane; this leads to

$$
\begin{aligned}
& b_{R \kappa}=-\frac{i}{2 \pi \sqrt{\kappa}} \Gamma(1+i \kappa) \int_{0}^{\infty} d k k^{-\frac{1}{2}-i \kappa}\left(a_{k} e^{\pi \kappa / 2}+a_{k}^{*} e^{-\pi \kappa / 2}\right), \\
& b_{R \kappa}^{*}=\frac{i}{2 \pi \sqrt{\kappa}} \Gamma(1-i \kappa) \int_{0}^{\infty} d k k^{-\frac{1}{2}+i \kappa}\left(a_{k} e^{-\pi \kappa / 2}+a_{k}^{*} e^{\pi \kappa / 2}\right) .
\end{aligned}
$$

A similar calculation for the $L$-wedge, in which $z=-(t+x)=e^{-(\tau+\xi)}$ gives

$$
\begin{aligned}
& b_{L \kappa}=\frac{i}{2 \pi \sqrt{\kappa}} \Gamma(1-i \kappa) \int_{0}^{\infty} d k k^{-\frac{1}{2}+i \kappa}\left(a_{k} e^{\pi \kappa / 2}+a_{k}^{*} e^{-\pi \kappa / 2}\right), \\
& b_{L \kappa}^{*}=-\frac{i}{2 \pi \sqrt{\kappa}} \Gamma(1+i \kappa) \int_{0}^{\infty} d k k^{-\frac{1}{2}-i \kappa}\left(a_{k} e^{-\pi \kappa / 2}+a_{k}^{*} e^{\pi \kappa / 2}\right) .
\end{aligned}
$$

Applying these operators to the bosonic Minkowski vacuum one establishes that

$$
\begin{aligned}
& e^{\pi \kappa / 2} b_{R \kappa}|0\rangle_{b}=e^{-\pi \kappa / 2} b_{L \kappa}^{*}|0\rangle_{b}=-\frac{i}{2 \pi \sqrt{\kappa}} \Gamma(1+i \kappa) \int_{0}^{\infty} d k k^{-\frac{1}{2}-i \kappa} a_{k}^{*}|0\rangle_{b}, \\
& e^{-\pi \kappa / 2} b_{R \kappa}^{*}|0\rangle_{b}=e^{\pi \kappa / 2} b_{L \kappa}|0\rangle_{b}=\frac{i}{2 \pi \sqrt{\kappa}} \Gamma(1-i \kappa) \int_{0}^{\infty} d k k^{-\frac{1}{2}+i \kappa} a_{k}^{*}|0\rangle_{b} .
\end{aligned}
$$

It follows that the Minkowski vacuum contains Rindler excitations, and that annihilation of an excitation in the right Rindler wedge has the same effect modulo a factor $e^{-\pi \kappa}$ as creation of one in the left Rindler wedge, and vice versa.

In order to relate the excitations of the fermion field in Minkowski and Rindler space, we proceed along similar lines, but with some slight modifications to keep track of the conformal and local Lorentz transformations relating the fields in the different observer frames. The starting point is provided by the Equation (39), in which we replace the Rindler spinor field $\psi_{+}$by its Minkowski counterpart (36). In the $R$-wedge of Rindler space, using the light-cone variable (45), we get

$$
\begin{aligned}
\beta_{R \kappa}=\frac{i}{4 \pi \kappa} \int_{0}^{\infty} d k \int_{0}^{\infty} \frac{d z}{z} z^{\frac{1}{2}+i \kappa} & {\left[\left(\frac{1}{2}-i \kappa-i k z\right) \alpha_{k} e^{-i k z}\right.} \\
+ & \left.\left(\frac{1}{2}-i \kappa+i k z\right) \alpha_{k}^{*} e^{i k z}\right], \\
\beta_{R \kappa}^{*}=-\frac{i}{4 \pi \kappa} \int_{0}^{\infty} d k \int_{0}^{\infty} \frac{d z}{z} z^{\frac{1}{2}-i \kappa} & {\left[\left(\frac{1}{2}+i \kappa-i k z\right) \alpha_{k} e^{-i k z}\right.} \\
+ & \left.\left(\frac{1}{2}+i \kappa+i k z\right) \alpha_{k}^{*} e^{i k z}\right] .
\end{aligned}
$$

Performing the integrations over $z$ in the compex plane this yields

$$
\begin{aligned}
& \beta_{R \kappa}=e^{-i \pi / 4} \Gamma\left(\frac{1}{2}+i \kappa\right) \int_{0}^{\infty} \frac{d k}{2 \pi} k^{-\frac{1}{2}-i \kappa}\left(\alpha_{k} e^{\pi \kappa / 2}+i \alpha_{k}^{*} e^{-\pi \kappa / 2}\right), \\
& \beta_{R \kappa}^{*}=e^{-i \pi / 4} \Gamma\left(\frac{1}{2}-i \kappa\right) \int_{0}^{\infty} \frac{d k}{2 \pi} k^{-\frac{1}{2}+i \kappa}\left(\alpha_{k} e^{-\pi \kappa / 2}+i \alpha_{k}^{*} e^{\pi \kappa / 2}\right) .
\end{aligned}
$$


Similarly in the $L$-wedge of Rindler space we obtain after the appropriate modifications

$$
\begin{aligned}
& \beta_{L \kappa}=e^{i \pi / 4} \Gamma\left(\frac{1}{2}-i \kappa\right) \int_{0}^{\infty} \frac{d k}{2 \pi} k^{-\frac{1}{2}+i \kappa}\left(\alpha_{k} e^{\pi \kappa / 2}-i \alpha_{k}^{*} e^{-\pi \kappa / 2}\right), \\
& \beta_{L \kappa}^{*}=e^{i \pi / 4} \Gamma\left(\frac{1}{2}+i \kappa\right) \int_{0}^{\infty} \frac{d k}{2 \pi} k^{-\frac{1}{2}-i \kappa}\left(\alpha_{k} e^{-\pi \kappa / 2}-i \alpha_{k}^{*} e^{\pi \kappa / 2}\right) .
\end{aligned}
$$

From this, we derive the following relations for the action on the fermionic Minkowski vacuum:

$$
\begin{aligned}
& e^{\pi \kappa / 2} \beta_{R \kappa}|0\rangle_{f}=-i e^{-\pi \kappa / 2} \beta_{L \kappa}^{*}|0\rangle_{f}=e^{i \pi / 4} \Gamma\left(\frac{1}{2}+i \kappa\right) \int_{0}^{\infty} \frac{d k}{2 \pi} k^{-\frac{1}{2}-i \kappa} \alpha_{k}^{*}|0\rangle_{f}, \\
& e^{-\pi \kappa / 2} \beta_{R \kappa}^{*}|0\rangle_{f}=i e^{\pi \kappa / 2} \beta_{L \kappa}|0\rangle_{f}=e^{i \pi / 4} \Gamma\left(\frac{1}{2}-i \kappa\right) \int_{0}^{\infty} \frac{d k}{2 \pi} k^{-\frac{1}{2}+i \kappa} \alpha_{k}^{*}|0\rangle_{f} .
\end{aligned}
$$

Again, the fermionic Minkowski vacuum contains fermionic Rindler excitations, with creation of one in the $R$-wedge being accompanied by annihilation of a similar one in the $L$-wedge, and vice versa.

As concerns correlation functions, in free field theories the only non-trivial correlators are two-point functions. In Minkowski space the elementary ones are

$$
{ }_{b}\left\langle 0\left|a_{k} a_{q}^{*}\right| 0\right\rangle_{b}={ }_{f}\left\langle 0\left|\alpha_{k} \alpha_{q}^{*}\right| 0\right\rangle_{f}=2 \pi \delta(k-q) .
$$

Using the results (49) and (53) in a recursive way [17], one establishes that the correlators of the Rindler fields in the Minkowski vacuum are

$$
{ }_{b}\left\langle 0\left|b_{R \kappa} b_{R \sigma}^{*}\right| 0\right\rangle_{b}=\frac{2 \pi \delta(\kappa-\sigma)}{1-e^{-2 \pi \kappa}}, \quad{ }_{f}\left\langle 0\left|\beta_{R \kappa} \beta_{R \sigma}^{*}\right| 0\right\rangle_{f}=\frac{2 \pi \delta(\kappa-\sigma)}{1+e^{-2 \pi \kappa}},
$$

with identical results for the correlators in the L-wedge. These relations imply the complementary identities

$$
{ }_{b}\left\langle 0\left|b_{R \kappa}^{*} b_{R \sigma}\right| 0\right\rangle_{b}=\frac{2 \pi \delta(\kappa-\sigma)}{e^{2 \pi \kappa}-1}, \quad{ }_{f}\left\langle 0\left|\beta_{R \kappa}^{*} \beta_{R \sigma}\right| 0\right\rangle_{f}=\frac{2 \pi \delta(\kappa-\sigma)}{e^{2 \pi \kappa}+1},
$$

stating that the occupation numbers of Rindler excitations in the Minkowski vacuum are of thermal Bose-Einstein and Fermi-Dirac type, corresponding to a state with temperature $T=1 / 2 \pi$; upon restoring the acceleration parameter by rescaling of the Rindler co-ordinates and momenta the temperature of Minkowski space observed in a Rindler frame with proper acceleration $a$ is $T=a / 2 \pi$. Details of the derivation are presented in Appendix B.

\section{Supersymmetry}

As we have seen, the supersymmetric theory has a unique Rindler hamiltonian (42), which is the square of the supercharge (43). In view of the results (56), it is easily seen to have a divergent Minkowski vacuum expectation value. It follows automatically that the Rindler supercharge does not annihilate the Minkowski vacuum: Rindler supersymmetry is broken by finite temperature effects in the Minkowski vacuum. In contrast, Minkowski supersymmetry generated by the supercharge (25) does annihilate the Minkowski vacuum, as stated by (27).

One way to deal with this situation it is to regularize the Rindler supercharge using a symmetric regulator function $g(\kappa, \sigma)=g(\sigma, \kappa)$ and define

$$
Q_{R g}=\int_{0}^{\infty} \frac{d \kappa}{2 \pi} \int_{0}^{\infty} \frac{d \sigma}{2 \pi} g(\kappa, \sigma)\left(b_{\kappa}^{*} \beta_{\sigma}+\beta_{\sigma}^{*} b_{\kappa}\right) .
$$


A straightforward calculation shows that

$$
Q_{R g}^{2}=\int_{0}^{\infty} \frac{d \kappa}{2 \pi} \int_{0}^{\infty} \frac{d \sigma}{2 \pi} g^{2}(\kappa, \sigma)\left(b_{\kappa}^{*} b_{\sigma}+\beta_{\kappa}^{*} \beta_{\sigma}\right) \equiv H_{R g},
$$

where

$$
g^{2}(\kappa, \sigma)=\int_{0}^{\infty} \frac{d \eta}{2 \pi} g(\kappa, \eta) g(\eta, \sigma) .
$$

This properly regularizes the Rindler supercharge and hamiltonian if $g$ is taken from a class of functions having a limit

$$
g(\kappa, \sigma) \rightarrow \sqrt{\kappa} \delta(\kappa-\sigma), \quad g^{2}(\kappa, \sigma) \rightarrow \kappa \delta(\kappa-\sigma) .
$$

With such a regulator, one easily finds that

$$
\begin{aligned}
& { }_{M}\left\langle 0\left|Q_{R g}^{2}\right| 0\right\rangle_{M}={ }_{M}\left\langle 0\left|H_{R g}\right| 0\right\rangle_{M} \\
& \quad=\int_{0}^{\infty} \frac{d \kappa}{2 \pi} g^{2}(\kappa, \kappa)\left(\frac{1}{e^{2 \pi \kappa}-1}+\frac{1}{e^{2 \pi \kappa}+1}\right)=\int_{0}^{\infty} \frac{d \kappa}{2 \pi} \frac{g^{2}(\kappa, \kappa)}{2 \sinh 2 \pi \kappa},
\end{aligned}
$$

which is a positive number for any acceptable regulator $g(\kappa, \sigma)$.

\section{Conformal Symmetries}

In addition to the hamiltonian and the supercharge, which are conserved for the theories in the Minkowski frame, one can construct an infinite set of conformal charges annihilating the vacuum state which define a continuum generalization of the Virasoro and super-Virasoro algebra.

The charges, which can be decomposed in contributions from bosons and from fermions, are labeled by the momentum variable $k$; for $k \geq 0$ they annihilate the Minkowski vacuum, being defined for a single boson and fermion field by

$$
\begin{aligned}
& L_{k}^{b}=\int_{0}^{\infty} \frac{d q}{2 \pi} \sqrt{q(k+q)} a_{q}^{*} a_{k+q}+\int_{0}^{k} \frac{d q}{4 \pi} \sqrt{q(k-q)} a_{q} a_{k-q}, \\
& L_{k}^{f}=\int_{0}^{\infty} \frac{d q}{2 \pi}\left(q+\frac{k}{2}\right) \alpha_{q}^{*} \alpha_{k+q}-\int_{0}^{k} \frac{d q}{4 \pi} q \alpha_{q} \alpha_{k-q},
\end{aligned}
$$

whilst those labeled by a negative momentum $-k$ are the hermitean conjugates $L_{-k}=L_{k}^{*}$. Introducing the general notation $a_{-k}=a_{k}^{*}, \alpha_{-k}=\alpha_{k}^{*}$, it is possible to write these expressions in a short-hand notation using normal ordering:

$$
L_{k}^{b}=\int_{-\infty}^{\infty} \frac{d q}{4 \pi} \sqrt{|q(q-k)|}: a_{q} a_{k-q}:, \quad L_{k}^{f}=\int_{-\infty}^{\infty} \frac{d q}{4 \pi}\left(-q+\frac{k}{2}\right): \alpha_{q} \alpha_{k-q}: .
$$

However, apart from the fact that the natural domain of the momentum labels $k, q$ is $[0, \infty)$, the normal ordering in Rindler space is ambiguous due to the mixing of creation an annhihilation operators by the Bogoliubov tranformations, as pointed out before. This is especially relevant, as we intend to compute correlation functions of Rindler operators in the Minkowski vacuum. Therefore, we prefer to write out the explicit expansions (62) rather than using the normal-ordered ones.

The operators (62) satisfy the commutation relations of the Virasoro algebra with a central charge:

$$
\begin{aligned}
& {\left[L_{k}^{b}, L_{l}^{b}\right]=(k-l) L_{k+l}^{b}+\frac{1}{12} k^{3} \delta(k+l),} \\
& {\left[L_{k}^{f}, L_{l}^{f}\right]=(k-l) L_{k+l}^{f}+\frac{1}{24} k^{3} \delta(k+l) .}
\end{aligned}
$$


The expressions for the central charges are easily verified by the standard procedure of evaluating the vacuum expectation value of the commutator

$$
\frac{1}{2}{ }_{b}\left\langle 0\left|\left[L_{k}^{b}, L_{l}^{b}\right]\right| 0\right\rangle_{b}={ }_{f}\left\langle 0\left|\left[L_{k}^{f}, L_{l}^{f}\right]\right| 0\right\rangle_{f}=\frac{1}{24} k^{3} \delta(k+l) .
$$

In supersymmetric Minkowski-frame theories, the Virasoro algebra can be extended by supercharges $G_{k}$, defined for a single boson plus fermion field and for $k \geq 0$ by

$$
G_{k}=\int_{0}^{\infty} \frac{d q}{2 \pi}\left(\sqrt{q} a_{q}^{*} \alpha_{k+q}+\sqrt{k+q} \alpha_{q}^{*} a_{k+q}\right)+\int_{0}^{k} \frac{d q}{2 \pi} \sqrt{q} a_{q} \alpha_{k-q} ;
$$

again for negative index $G_{-k}=G_{k}^{*}$. In a theory with a single boson and fermion the complete set of (anti-)commutation relations with

$$
L_{k}=L_{k}^{b}+L_{k}^{f}
$$

is found to have the standard super-Virasoro form

$$
\begin{aligned}
& {\left[L_{k}, L_{l}\right]=(k-l) L_{k+l}+\frac{1}{8} k^{3} \delta(k+l),} \\
& {\left[L_{k}, G_{l}\right]=\left(\frac{k}{2}-l\right) G_{k+l},} \\
& \left\{G_{k}, G_{l}\right\}=2 L_{k+l}+\frac{1}{2} k^{2} \delta(k+l) .
\end{aligned}
$$

Note that $L_{0}=H$ is the hamiltonian (24) and $G_{0}=Q$ is the supercharge (25). They define a closed anomaly-free subalgebra of the super-Virasoro algebra (66). Also note that their vacuum expectations vanish:

$$
\begin{aligned}
\forall k \geq 0: \quad L_{k}|0\rangle_{M}=0 \quad \Rightarrow \quad{ }_{M}\left\langle 0\left|L_{k}\right| 0\right\rangle_{M}={ }_{M}\left\langle 0\left|L_{-k}\right| 0\right\rangle_{M}=0, \\
G_{k}|0\rangle_{M}=0 \quad \Rightarrow \quad{ }_{M}\left\langle 0\left|G_{k}\right| 0\right\rangle_{M}={ }_{M}\left\langle 0\left|G_{-k}\right| 0\right\rangle_{M}=0 .
\end{aligned}
$$

The same construction also works in the Rindler frame, where in the $R$-wedge of Rindler space

$$
\begin{aligned}
& L_{R \kappa}^{b} \equiv \int_{0}^{\infty} \frac{d \sigma}{2 \pi} \sqrt{\sigma(\kappa+\sigma)} b_{R \sigma}^{*} b_{R \kappa+\sigma}+\int_{0}^{\kappa} \frac{d \sigma}{4 \pi} \sqrt{\sigma(\kappa-\sigma)} b_{R \sigma} b_{R \kappa-\sigma}, \\
& L_{R \kappa}^{f}=\int_{0}^{\infty} \frac{d \sigma}{2 \pi}\left(\sigma+\frac{\kappa}{2}\right) \beta_{R \sigma}^{*} \beta_{R \kappa+\sigma}-\int_{0}^{\kappa} \frac{d \sigma}{4 \pi} \sigma \beta_{R \sigma} \beta_{R \kappa-\sigma}, \\
& G_{R \kappa}=\int_{0}^{\infty} \frac{d \sigma}{2 \pi}\left(\sqrt{\sigma} b_{R \sigma}^{*} \beta_{R \kappa+\sigma}+\sqrt{\kappa+\sigma} \beta_{R \sigma}^{*} b_{R \kappa+\sigma}\right)+\int_{0}^{\kappa} \frac{d \sigma}{2 \pi} \sqrt{\sigma} b_{R \sigma} \beta_{R \kappa-\sigma},
\end{aligned}
$$

with analogous definitions in the $L$-wedge. The essential difference with the Minkowski charges, is that the Rindler operators (68) admit a non-vanishing expectation value in the Minkowski frame:

$$
\begin{aligned}
{ }_{M}\left\langle 0\left|L_{R \kappa}^{b}\right| 0\right\rangle_{M} & =\delta(\kappa) \int_{0}^{\infty} d \sigma \frac{\sigma}{e^{2 \pi \sigma}-1}=\frac{1}{24} \delta(\kappa), \\
{ }_{M}\left\langle 0\left|L_{R \kappa}^{f}\right| 0\right\rangle_{M} & =\delta(\kappa) \int_{0}^{\infty} d \sigma \frac{\sigma}{e^{2 \pi \sigma}+1}=\frac{1}{48} \delta(\kappa),
\end{aligned}
$$

whilst in the superconformal case

$$
{ }_{M}\left\langle 0\left|G_{R \kappa}\right| 0\right\rangle_{M}=0 .
$$


Although one could remove these vacuum expectation values by shifting the ground-state energy, this would introduce an extra contribution to the central charge, which is generally not desirable. The singular nature of the expectation value of the hamiltonian $H=L_{0}$, in practice, requires regularization, as we discussed in Section 5.

\section{Ghosts and Local Conformal Symmetry}

As is well-known, the line element in any topologically trivial 2-D space-time can be cast in the form (1) by appropriate co-ordinate transformations. As 2-D gravity is conformally invariant, but non-dynamical, one can therefore interpret the boson and fermion field theories discussed above as the gauge-fixed version of a gravitational theory with local conformal symmetry. This is the key to a consistent quantum-theory of strings and superstrings [4]. Local conformal symmetry turns the generators of the Virasoro algebra into operators imposing first-class constraints:

$$
\forall k>0: \quad L_{k}|p h y s\rangle=0 .
$$

These conditions are consistent only if the anomaly vanishes, but the anomaly now includes a contribution from the Faddeev-Popov ghosts introduced by quantization of the gravitational background as well [20].

In the sector of left-moving fields the dynamics of the anti-commuting gravity ghosts $(C, D)$ follows from the action

$$
S_{g}=i \int_{M} d^{2} x D\left(\partial_{t}-\partial_{x}\right) C
$$

Following standard procedures, one derives the hamiltonian

$$
H_{g}=i \int_{-\infty}^{\infty} d x D \partial_{x} C
$$

supplemented by the equal-time anti-commutation relations

$$
\{C(t, x), D(t, y)\}=\delta(x-y)
$$

From the mode expansions

$$
\begin{aligned}
& C(t, x)=\int_{0}^{\infty} \frac{d k}{2 \pi}\left(c_{k} e^{-i k(t+x)}+c_{k}^{*} e^{i k(t+x)}\right), \\
& D(t, x)=\int_{0}^{\infty} \frac{d k}{2 \pi}\left(d_{k} e^{-i k(t+x)}+d_{k}^{*} e^{i k(t+x)}\right),
\end{aligned}
$$

we derive the anti-commutators of the mode operators:

$$
\left\{c_{k}, d_{q}^{*}\right\}=2 \pi \delta(k-q), \quad\left\{d_{k}, c_{q}^{*}\right\}=2 \pi \delta(k-q) .
$$

The corresponding Virasoro generators for $k \geq 0$ are

$$
L_{k}^{g}=\int_{0}^{\infty} \frac{d q}{2 \pi}\left[(q-k) c_{q}^{*} d_{k+q}+(q+2 k) d_{q}^{*} c_{k+q}\right]-\int_{0}^{k} \frac{d q}{2 \pi}(q+k) c_{q} d_{k-q},
$$

with $L_{-k}^{g}=L_{k}^{g *}$. The ghost Virasoro algebra then becomes

$$
\left[L_{k}^{g}, L_{l}^{g}\right]=(k-l) L_{k+l}^{g}-\frac{13}{6} k^{3} \delta(k+l) .
$$


For a theory with $N_{b}$ massless scalars and $N_{f}$ massless chiral Majorana fermions the full first-class contraints then are

$$
\forall k>0: \quad L_{k}|p h y s\rangle=0, \quad L_{k}=\sum_{i=1}^{N_{b}} L_{k}^{b i}+\sum_{j=1}^{N_{f}} L_{k}^{f j}+L_{k}^{g},
$$

with the algebra

$$
\left[L_{k}, L_{l}\right]=(k-l) L_{k+l}+\frac{\left(2 N_{b}+N_{f}-52\right)}{24} k^{3} \delta(k+l) .
$$

Thus, the central charge vanishes provided

$$
2 N_{b}+N_{f}=52
$$

The analysis in the right-moving sector proceeds entirely analogously.

In the case of a supersymmetric theory of massless scalars and femions the local conformal symmetry can be extended to local superconformal symmetry. Such a theory is effectively a gauge-fixed 2-D supergravity theory and requires an additional set of commuting supersymmetry ghosts $(S, U)$ with action

$$
S_{s g}=\int_{M} d^{2} x U\left(\partial_{t}-\partial_{x}\right) S .
$$

The associated hamiltonian and equal-time commutation relations are

$$
H_{s g}=\int_{-\infty}^{\infty} d x U \partial_{x} S, \quad[S(t, x), U(t, y)]=i \delta(x-y) .
$$

In this case, we take the plane-wave expansions to be

$$
\begin{aligned}
& S(t, x)=\int_{0}^{\infty} \frac{d k}{2 \pi}\left(s_{k} e^{-i k(t+x)}+s_{k}^{*} e^{i k(t+x)}\right), \\
& U(t, x)=-i \int_{0}^{\infty} \frac{d k}{2 \pi}\left(u_{k} e^{-i k(t+x)}-u_{k}^{*} e^{i k(t+x)}\right),
\end{aligned}
$$

which results in mode commutation relations

$$
\left[s_{k}, u_{q}^{*}\right]=2 \pi \delta(k-q), \quad\left[u_{k}, s_{q}^{*}\right]=2 \pi \delta(k-q) .
$$

The super-Virasoro operators of the full set of ghosts $(C, D)$ and superghosts $(S, U)$ then is defined by (76) and for $k \geq 0$ :

$$
\begin{aligned}
L_{k}^{s g}= & \int_{0}^{\infty} \frac{d q}{2 \pi}\left[\left(q-\frac{k}{2}\right) s_{q}^{*} u_{k+q}+\left(q+\frac{3 k}{2}\right) u_{q}^{*} s_{k+q}\right] \\
& -\int_{0}^{k} \frac{d q}{2 \pi}\left(q+\frac{k}{2}\right) s_{q} u_{k-q}, \\
G_{k}^{s g}= & \int_{0}^{\infty} \frac{d q}{2 \pi}\left[s_{q}^{*} b_{k+q}+b_{q}^{*} s_{k+q}+(q+3 k) u_{q}^{*} c_{k+q}+(q-2 k) c_{q}^{*} u_{k+q}\right] \\
& +\int_{0}^{k} \frac{d q}{2 \pi}\left[s_{q} b_{k-q}-(q+2 k) c_{q} u_{k-q}\right],
\end{aligned}
$$


with $L_{-k}^{s g}=L_{k}^{s g *}$ and $G_{-k}^{s g}=G_{k}^{s g *}$. The complete super-Virasoro algebra of the ghosts is given by $(77)$ and

$$
\begin{aligned}
& {\left[L_{k}^{s g}, L_{l}^{s g}\right]=(k-l) L_{k+l}^{s g}+\frac{11}{12} k^{3} \delta(k+l),} \\
& \left\{G_{k}^{s g}, G_{l}^{s g}\right\}=2\left(L_{k+l}^{g}+L_{k+l}^{s g}\right)-5 k^{2} \delta(k+l) .
\end{aligned}
$$

With these results a 2-D supersymmetric theory of $N$ massless scalars and fermions has local superconformal symmetry if

$$
\forall k>0: \quad L_{k}|p h y s\rangle=G_{k}|p h y s\rangle=0,
$$

where

$$
L_{k}=\sum_{i=1}^{N}\left(L_{k}^{b i}+L_{k}^{f i}\right)+L_{k}^{g}+L_{k}^{s g}, \quad G_{k}=\sum_{i=1}^{N} G_{k}^{i}+G_{k}^{s g}
$$

with the algebra

$$
\begin{aligned}
& {\left[L_{k}, L_{l}\right]=(k-l) L_{k+l}+\frac{(N-10)}{8} k^{3} \delta(k+l),} \\
& {\left[L_{k}, G_{l}\right]=\left(\frac{k}{2}-l\right) G_{k+l},} \\
& \left\{G_{k}, G_{l}\right\}=2 L_{k+l}+\frac{(N-10)}{2} k^{2} \delta(k+l) .
\end{aligned}
$$

Thus all of the superconformal anomalies vanish for $N=10$. Of course, all results derived here are in agreement with corresponding string and superstring theories in the limit of infinite strings.

\section{Conformal and Superconformal Ghosts in Rindler Space}

The local conformal and superconformal symmetries arising in a gauge-fixed 2-D gravity or supergravity theory with scalar and spinor matter can be extended to the Rindler frame. It requires solving the ghost and superghost field equations in Rindler space, and finding the appropriate Bogoliubov tranformations.

Consider a theory in an arbitrary 2-D space-time with reparametrization and locally Lorentz-invariant action

$$
S[F, G]=\int d^{2} x e F^{a_{1} \ldots a_{n+1}} e_{a_{n+1}}^{\mu} D_{\mu} G_{a_{1} \ldots a_{n}} .
$$

Here, $(F, G)$ is a system of commuting or anti-commuting local Lorentz tensors or (chiral) spinor-tensors of rank $(n, n+1), e_{a}^{\mu}$ is the inverse 2-bein field as explained in Appendix A; $D_{\mu}$ is the Lorentz-covariant derivative with spin connection $\omega_{\mu b}^{a}$ defined in (A7) and $e=\operatorname{det} e_{\mu}^{a}=\sqrt{-g}$. Consider only purely left- or right-handed components associated with purely self-dual or anti-self-dual tensors:

$$
G_{a_{1} \ldots a_{n}}= \pm \varepsilon_{a_{1} b} G_{a_{2} \ldots a_{n}}^{b}=\ldots= \pm \varepsilon_{a_{n} b} G_{a_{1} \ldots a_{n-1}},
$$

and similarly for $F^{a_{1} \ldots a_{n+1}}$. Fixing the Minkowski gauge, the action (90) can be decomposed into actions of the type (71) or (81) for single pairs of components $\left(F_{M}^{(n+1)}, G_{M}^{(n)}\right)$ with gauge-fixed action (considering left-movers for definiteness)

$$
S_{M}^{(n)}=\int_{M} d^{2} x F_{M}^{(n+1)}\left(\partial_{t}-\partial_{x}\right) G_{M}^{(n)}
$$


When transforming the action from the Minkowski to the Rindler frame by the transformations (3) with $a=1$ and following the same arguments, as described for spinor components in Appendix A, we obtain a Rindler-frame action in the $R$-wedge of Rindler space

$$
S_{R}^{(n)}=\int_{R} d \tau d \xi\left[F_{R}^{(n+1)}\left(\partial_{\tau}-\partial_{\xi}\right) G_{R}^{(n)}\right]
$$

where the field components have been transformed from the Minkowski to the Rindler frame by an extension of (36):

$$
\begin{aligned}
& F_{R}^{(n+1)}(\tau, \xi)=e^{(n+1)(\tau+\xi)} F_{M}^{(n+1)}[t(\tau, \xi), x(\tau, \xi)], \\
& G_{R}^{n}(\tau, \xi)=e^{-n(\tau+\xi)} G_{M}^{n}[t(\tau, \xi), x(\tau, \xi)] .
\end{aligned}
$$

Actually, $n$ can be integer or half-integer, depending on whether $(F, G)$ are tensors or spinor-tensors. The aim is now to connect the Rindler plane-wave expansions in the $R$-wedge

$$
\begin{aligned}
& F_{R}^{(n+1)}=\int_{0}^{\infty} \frac{d \kappa}{2 \pi}\left(f_{R \kappa} e^{-i \kappa(\tau+\xi)}+f_{R \kappa}^{*} e^{i \kappa(\tau+\xi)}\right) \\
& G_{R}^{(n)}=\int_{0}^{\infty} \frac{d \kappa}{2 \pi}\left(g_{R \kappa} e^{-i \kappa(\tau+\xi)}+g_{R \kappa}^{*} e^{i \kappa(\tau+\xi)}\right),
\end{aligned}
$$

with the Minkowski ones

$$
\begin{aligned}
& F_{M}^{(n+1)}=\int_{0}^{\infty} \frac{d k}{2 \pi}\left(f_{k} e^{-i k(t+x)}+f_{\kappa}^{*} e^{i \kappa(t+x)}\right) \\
& G_{M}^{(n)}=\int_{0}^{\infty} \frac{d k}{2 \pi}\left(g_{k} e^{-k(t+x)}+g_{\kappa}^{*} e^{i k(t+x)}\right) .
\end{aligned}
$$

Inverting the Equation (95) while using the light-cone variable $z$ defined in (45):

$$
\begin{aligned}
f_{R \kappa} & =\frac{i}{2 \kappa} \int_{-\infty}^{\infty} d \xi e^{i \kappa(\tau+\xi)} \overleftrightarrow{\partial}_{\tau} F_{R}^{(n+1)}(\tau, \xi) \\
& =\frac{i}{2 \kappa} \int_{0}^{\infty} \frac{d z}{z} z^{-n-1+i \kappa}\left(-i \kappa+n+1+z \partial_{z}\right) F_{M}^{(n+1)}(z) \\
g_{R \kappa} & =\frac{i}{2 \kappa} \int_{-\infty}^{\infty} d \xi e^{i \kappa(\tau+\xi)} \overleftrightarrow{\partial}_{\tau} G_{R}^{(n)}(\tau, \xi) \\
& =\frac{i}{2 \kappa} \int_{0}^{\infty} \frac{d z}{z} z^{n+i \kappa}\left(-i \kappa-n+z \partial_{z}\right) G_{M}^{(n)}(z)
\end{aligned}
$$

Substitutions of the Minkowski-frame plane-wave solutions yields

$$
\begin{aligned}
& f_{R \kappa}=i^{(n+1)} \Gamma(n+1+i \kappa) \int_{0}^{\infty} \frac{d k}{2 \pi} k^{-n-1-i \kappa}\left((-1)^{n+1} e^{\pi \kappa / 2} f_{k}+e^{-\pi \kappa / 2} f_{k}^{*}\right), \\
& g_{R \kappa}=i^{n} \Gamma(-n+i \kappa) \int_{0}^{\infty} \frac{d k}{2 \pi} k^{n-i \kappa}\left(e^{\pi \kappa / 2} g_{k}+(-1)^{n} e^{-\pi \kappa / 2} g_{k}^{*}\right),
\end{aligned}
$$


with conjugates

$$
\begin{aligned}
& f_{R \kappa}^{*}=i^{(n+1)} \Gamma(n+1-i \kappa) \int_{0}^{\infty} \frac{d k}{2 \pi} k^{-n-1+i \kappa}\left((-1)^{n+1} e^{-\pi \kappa / 2} f_{k}+e^{\pi \kappa / 2} f_{k}^{*}\right), \\
& g_{R \kappa}^{*}=i^{n} \Gamma(-n-i \kappa) \int_{0}^{\infty} \frac{d k}{2 \pi} k^{n+i \kappa}\left(e^{-\pi \kappa / 2} g_{k}+(-1)^{n} e^{\pi \kappa / 2} g_{k}^{*}\right) .
\end{aligned}
$$

In the $L$-wedge of Rindler space, one finds by analogous calculations for the left-moving fields

$$
\begin{aligned}
& f_{L \kappa}=i^{(n+1)} \Gamma(n+1-i \kappa) \int_{0}^{\infty} \frac{d k}{2 \pi} k^{-n-1+i \kappa}\left(e^{\pi \kappa / 2} f_{k}+(-1)^{n+1} e^{-\pi \kappa / 2} f_{k}^{*}\right), \\
& g_{L \kappa}=i^{n} \Gamma(-n-i \kappa) \int_{0}^{\infty} \frac{d k}{2 \pi} k^{n+i \kappa}\left((-1)^{n} e^{\pi \kappa / 2} g_{k}+e^{-\pi \kappa / 2} g_{k}^{*}\right) .
\end{aligned}
$$

and conjugates

$$
\begin{aligned}
& f_{L \kappa}^{*}=i^{(n+1)} \Gamma(n+1+i \kappa) \int_{0}^{\infty} \frac{d k}{2 \pi} k^{-n-1-i \kappa}\left(e^{-\pi \kappa / 2} f_{k}+(-1)^{n+1} e^{\pi \kappa / 2} f_{k}^{*}\right), \\
& g_{L \kappa}^{*}=i^{n} \Gamma(-n+i \kappa) \int_{0}^{\infty} \frac{d k}{2 \pi} k^{n-i \kappa}\left((-1)^{n} e^{-\pi \kappa / 2} g_{k}+e^{\pi \kappa / 2} g_{k}^{*}\right) .
\end{aligned}
$$

Upon application to the Minkowski vacuum, it follows that

$$
\begin{array}{cc}
e^{\pi \kappa / 2} f_{R \kappa}|0\rangle_{M}=(-1)^{n+1} e^{-\pi \kappa / 2} f_{L \kappa}^{*}|0\rangle_{M}, & e^{-\pi \kappa / 2} f_{R \kappa}^{*}|0\rangle_{M}=(-1)^{n+1} e^{\pi \kappa / 2} f_{L \kappa}|0\rangle_{M}, \\
(-1)^{n} e^{\pi \kappa / 2} g_{R \kappa}|0\rangle_{M}=e^{-\pi \kappa / 2} g_{L \kappa}^{*}|0\rangle_{M}, & (-1)^{n} e^{-\pi \kappa / 2} g_{R \kappa}^{*}|0\rangle_{M}=e^{\pi \kappa / 2} g_{L \kappa}|0\rangle_{M} .
\end{array}
$$

Applying these results to the ghost system (71), which fits the system with $n=1[4,20,21]$, and obeying the anti-commutation rules

$$
\left\{c_{R \kappa}, d_{R \sigma}^{*}\right\}=\left\{c_{L \kappa}, d_{L \sigma}^{*}\right\}=\left\{d_{R \kappa}, c_{R \sigma}^{*}\right\}=\left\{d_{L \kappa}, c_{L \sigma}^{*}\right\}=2 \pi \delta(\kappa-\sigma),
$$

we can compute the two-point correlations of the Rindler ghosts in the Minkowski vacuum:

$$
\begin{aligned}
& { }_{M}\left\langle 0\left|b_{R \sigma} c_{R \kappa}^{*}\right| 0\right\rangle_{M}={ }_{M}\left\langle 0\left|c_{R \sigma} b_{R \kappa}^{*}\right| 0\right\rangle_{M}=\frac{2 \pi \delta(\kappa-\sigma)}{1-e^{-2 \pi \kappa}}, \\
& { }_{M}\left\langle 0\left|c_{R \kappa}^{*} b_{R \sigma}\right| 0\right\rangle_{M}={ }_{M}\left\langle 0\left|b_{R \kappa}^{*} c_{R \sigma}\right| 0\right\rangle_{M}=-\frac{2 \pi \delta(\kappa-\sigma)}{e^{2 \pi \kappa}-1} .
\end{aligned}
$$

Note that these results are independent of the value of $n$, but do reflect the ghost statistics leading to the minus sign in the last expression.

Finally, we turn to the commuting superghosts $(S, U)$. The analysis proceeds parallel to the ghost system $(C, D)$, except that the appropriate value Lorentz and conformal weight is $n=1 / 2[4,22]$ and the anti-commutation rules are replaced by commutation rules. The relevant results are

$$
\begin{array}{cc}
e^{\pi \kappa / 2} u_{R \kappa}|0\rangle_{M}=(-1)^{n+1} e^{-\pi \kappa / 2} u_{L \kappa}^{*}|0\rangle_{M}, & e^{-\pi \kappa / 2} u_{R \kappa}^{*}|0\rangle_{M}=(-1)^{n+1} e^{\pi \kappa / 2} u_{L \kappa}|0\rangle_{M}, \\
(-1)^{n} e^{\pi \kappa / 2} s_{R \kappa}|0\rangle_{M}=e^{-\pi \kappa / 2} s_{L \kappa}^{*}|0\rangle_{M}, & (-1)^{n} e^{-\pi \kappa / 2} s_{R \kappa}^{*}|0\rangle_{M}=e^{\pi \kappa / 2} s_{L \kappa}|0\rangle_{M},
\end{array}
$$

supplemented by the commutation rules

$$
\left[s_{R \kappa}, u_{R \sigma}^{*}\right]=\left[s_{L \kappa}, u_{L \sigma}^{*}\right]=\left[u_{R \kappa}, s_{R \sigma}^{*}\right]=\left[u_{L \kappa}, s_{L \sigma}^{*}\right]=2 \pi \delta(\kappa-\sigma) .
$$


From these equations, one derives the Minkowski two-point functions for the superghosts:

$$
\begin{aligned}
& { }_{M}\left\langle 0\left|u_{R \sigma} s_{R \kappa}^{*}\right| 0\right\rangle_{M}={ }_{M}\left\langle 0\left|s_{R \sigma} u_{R \kappa}^{*}\right| 0\right\rangle_{M}=\frac{2 \pi \delta(\kappa-\sigma)}{1+e^{-2 \pi \kappa}}, \\
& { }_{M}\left\langle 0\left|s_{R \kappa}^{*} u_{R \sigma}\right| 0\right\rangle_{M}={ }_{M}\left\langle 0\left|u_{R \kappa}^{*} s_{R \sigma}\right| 0\right\rangle_{M}=-\frac{2 \pi \delta(\kappa-\sigma)}{e^{2 \pi \kappa}+1} .
\end{aligned}
$$

\section{Local Conformal Invariance in Rindler Space}

With the results (104) and (107), we can now compute the Minkowski expectation values of the (super-)Virasoro operators, including the ghost contributions required for local conformal and superconformal invariance. First, the results (68) are supplemented for $\kappa \geq 0$ by

$$
\begin{aligned}
L_{R \kappa}^{g}= & \int_{0}^{\infty} \frac{d \sigma}{2 \pi}\left[(\sigma-\kappa) c_{\sigma}^{*} d_{\kappa+\sigma}+(\sigma+2 \kappa) d_{\sigma}^{*} c_{\kappa+\sigma}\right]-\int_{0}^{\kappa} \frac{d \sigma}{2 \pi}(\sigma+\kappa) c_{\sigma} d_{\kappa-\sigma}, \\
L_{R \kappa}^{s g}= & \int_{0}^{\infty} \frac{d \sigma}{2 \pi}\left[\left(\sigma-\frac{\kappa}{2}\right) s_{\sigma}^{*} u_{\kappa+\sigma}+\left(\sigma+\frac{3 \kappa}{2}\right) u_{\sigma}^{*} s_{\kappa+\sigma}\right] \\
& -\int_{0}^{\kappa} \frac{d \sigma}{2 \pi}\left(\sigma+\frac{\kappa}{2}\right) s_{\sigma} u_{\kappa-\sigma}, \\
G_{R \kappa}^{s g}= & \int_{0}^{\infty} \frac{d \sigma}{2 \pi}\left[s_{\sigma}^{*} b_{\kappa+\sigma}+b_{\sigma}^{*} s_{\kappa+\sigma}+(\sigma+3 \kappa) u_{\sigma}^{*} c_{\kappa+\sigma}+(\sigma-2 \kappa) c_{\sigma}^{*} u_{\kappa+\sigma}\right] \\
& +\int_{0}^{\kappa} \frac{d \sigma}{2 \pi}\left[s_{\sigma} b_{\kappa-\sigma}-(\sigma+2 \kappa) c_{\sigma} u_{\kappa-\sigma}\right],
\end{aligned}
$$

with $\left(L_{R}^{g}\right)_{-\kappa}=L_{R \kappa^{\prime}}^{g *}\left(L_{R}^{s g}\right)_{-\kappa}=L_{R \kappa}^{s g *}$ and $\left(G_{R}^{s g}\right)_{-\kappa}=G_{R \kappa}^{s g *}$. Together with the operators (68) for each scalar and spinor field, they define the same algebra (89) in Rindler space. Thus, the first-class constraints requiring the cancellation of the anomalies are also satisfied for $2 N_{b}+N_{f}=52$ bosons and fermions coupled to 2-D conformal gravity and $N_{b}=N_{f}=10$ bosons and fermions coupled to 2-D conformal supergravity.

As in the case of global conformal invariance, the Minkowski expectation values of the conformal charges are modified due to finite temperature effects. With the help of Equations (105) and (107), the results (69) are now extended by

$$
{ }_{M}\left\langle 0\left|L_{R \kappa}^{g}\right| 0\right\rangle_{M}=-\frac{1}{12} \delta(\kappa), \quad{ }_{M}\left\langle 0\left|L_{R \kappa}^{s g}\right| 0\right\rangle_{M}=-\frac{1}{24} \delta(\kappa) .
$$

For the full Virasoro charges, simultaneously requiring the cancellation of the anomalies:

$$
\begin{aligned}
& { }_{M}\left\langle 0\left|\left(N_{b} L_{R \kappa}^{b}+N_{f} L_{R \kappa}^{f}+L_{R \kappa}^{g}\right)\right| 0\right\rangle_{M}=\delta(\kappa), \\
& { }_{M}\left\langle 0\left|\left(N_{b} L_{R \kappa}^{b}+N_{f} L_{R \kappa}^{f}+L_{R \kappa}^{g}+L_{R \kappa}^{s g}\right)\right| 0\right\rangle_{M}=\frac{1}{2} \delta(\kappa) .
\end{aligned}
$$

These expectation values, restricted to $\kappa=0$, do not contradict the first-class constraints, at the same time showing that the full hamiltonian in Rindler space has a non-vanishing Minkowski expectation value as a result of finite-temperature correlations (104) and (107). As discussed before, a regularization procedure is required in order to deal with the $\delta$-function singularity of the expectation values in applications. 


\section{Conclusions}

Two-dimensional conformal field theories can be formulated in an inertial frame with a Minkowski metric, or in a uniformly accelerated frame with a local conformal Rindler metric defined by $\rho=e^{ \pm a \xi}$; here, the sign differentiates between the right and left wedge of Rindler space. Thus, in each wedge one obtains two different descriptions of the same theory. The switch between the frames not only changes the co-ordinates, but Fock-space quantization can proceed parallel in both frames, provided an appropriate conformal transformation is applied to the field components as well. The Fock spaces in the two frames are related by Bogoliubov transformations of the mode operators, effectively giving rise to finite-temperature correlations of the Rindler modes for states build on the Minkowski vacuum, which is the true ground state of the theory. The temperature is proportional to the acceleration, an effect well-known from the original work of Unruh, Davies, Wald, Fulling, and others for accelerated frames in Minkowski space, and the work of Hawking in the context of black hole space-times.

The switch to the Rindler frame not only creates an effective finite-temperature description, but changes the construction and action of symmetry operators, like conformal and supersymmetry charges on the ground state. The naive conformal and supersymmetries in the Rindler frame are broken by finite-temperature effects in the Minkowski vacuum, but the underlying Minkowski symmetries are still present, even though hidden by the Bogoliubov transformations.

An additional, different issue is the breaking of conformal and supersymmetries by anomalies, extensively studied in the context of string theory. In the field-theory view taken here, they break the local conformal and supersymmetry arising from coupling conformal matter to conformally invariant gravity or supergravity. Thus, consistent coupling to gravity or supergravity is only possible in the case of specific critical theories with a strongly limited spectrum of boson and fermion matter fields, reflecting the critical dimensions of quantum string theories. This is also relevant as the Minkowski-frame and Rindler-frame formulations of massless QFT's are related by a conformal transformation; if this transformation is jeopardized by the conformal anomaly in theories with a non-critical spectrum, it may require compensating Wess-Zumino type dynamics [23], e.g., involving a Liouville field.

The analysis that is presented here is strictly for massless free fields in two-dimensional space-time. To deal with two-dimensional conformal fields theories with non-trivial interactions is an interesting next step to the work presented here, as is the extension to higher-dimensional field theories. Results establishing the Unruh effect in more general field theories, mostly scalar fields, can be found in the literature, see [17] and references therein. However, the precise procedure and effects of conformal and supersymmetry transformations involving fields of non-zero spin in those cases remain to be established.

Funding: This research received no external funding.

Acknowledgments: I thank Bert Schellekens for a preliminary reading of the manuscript.

Conflicts of Interest: The author declares no conflict of interest.

\section{Appendix A. 2-D Spinor Conventions}

In this appendix and in the main body of the paper we denote by greek indices $\mu, v, \ldots$ vector en tensor components in an arbitrary space-time manifold with metric $g_{\mu \nu}$; latin indices $a, b, \ldots$ refer to vector and tensor components in a tangent Minkowski space with mertric $\eta_{a b}$. The Majorana representation of the Dirac algebra in 2-dimensional Minkowski space is defined in terms of the $2 \times 2$ Pauli matrices as follows:

$$
\gamma^{0}=i \sigma_{2}, \quad \gamma^{1}=\sigma_{1}, \quad \gamma_{3}=\gamma^{0} \gamma^{1}=\sigma_{3}
$$


In 2-dimensional Minkowski space a Dirac spinor is a 2-component object transforming under Lorentz transformations with parameter $\omega^{a b}=\omega \varepsilon^{a b}$ as

$$
\lambda^{\prime}=e^{\omega^{a b} \sigma_{a b} / 2} \lambda=e^{\omega \gamma_{3} / 2} \lambda, \quad \sigma_{a b}=\frac{1}{4}\left[\gamma_{a}, \gamma_{b}\right]=-\frac{1}{2} \varepsilon_{a b} \gamma_{3}
$$

Dirac-conjugate spinors are defined by $\bar{\lambda}=\lambda^{\dagger} \gamma_{0}$. The charge-conjugation operator is $C=-C^{T}=\gamma_{0}$ with the defining property

$$
C \gamma^{a} C^{-1}=-\gamma^{a T}
$$

A self-conjugate Majorana spinor $\lambda=\lambda^{c}=C \bar{\lambda}^{T}=\lambda^{*}$ is therefore a spinor with real components:

$$
\lambda=\lambda^{c}=\left[\begin{array}{c}
\lambda_{+} \\
\lambda_{-}
\end{array}\right], \quad \lambda_{ \pm}^{*}=\lambda_{ \pm}
$$

The subscripts \pm define the eigenvalues under the chiral operator $\gamma_{3}$. Evidently

$$
\bar{\lambda}=\lambda^{T} C
$$

In a general 2-D space-time the spinors are defined as representations of the local Lorentz group, transforming like (A2) with a space-time dependent parameter $\omega(x)$. At the same time a spinor transforms like a scalar under diffeomorphisms: $\lambda^{\prime}\left(x^{\prime}\right)=\lambda(x)$. To construct the Dirac-operator one uses the 2-bein fields $e_{\mu}^{a}(x)$ such that the metric is factorized as

$$
g_{\mu \nu}=e_{\mu}^{a} e_{v}^{b} \eta_{a b}
$$

In terms of the 2-bein fields and their inverse components $e_{a}^{\mu}$ the spin connection, acting as the gauge field for local Lorentz transformations, is defined by an extension of the metric postulate

$$
\nabla_{\mu} e_{v}^{a}=\partial_{\mu} e_{v}^{a}-\Gamma_{\mu \nu}^{\lambda} e_{\lambda}^{a}=\omega_{\mu b}^{a} e_{v}^{b}
$$

implying that

$$
\begin{gathered}
\omega_{\mu}^{a b}=-\omega_{\mu}^{b a}=\frac{1}{2}\left[e^{v a}\left(\partial_{\nu} e_{\mu}^{b}-\partial_{\mu} e_{v}^{b}\right)-e^{v b}\left(\partial_{\nu} e_{\mu}^{a}-\partial_{\mu} e_{v}^{a}\right)\right. \\
\left.+e^{\lambda a} e^{v b} e_{\mu c}\left(\partial_{\lambda} e_{v}^{c}-\partial_{\nu} e_{\lambda}^{c}\right)\right] .
\end{gathered}
$$

In terms of these objects the full space-time and Lorentz covariant derivative of a spinor field is

$$
D_{\mu} \lambda=\left(\partial_{\mu}-\frac{1}{2} \omega_{\mu}^{a b} \sigma_{a b}\right) \lambda
$$

the Dirac operator being defined by $\not D=\gamma^{a} e_{a}^{\mu} D_{\mu}$.

Next turn to the special class of conformally flat metrics (1). For these metrics (in a hybrid notation)

$$
e_{\mu}^{a}=\rho \delta_{\mu \prime}^{a}, \quad e_{a}^{\mu}=\frac{1}{\rho} \delta_{a}^{\mu}
$$

It follows that the components of the spin connection are

$$
\omega_{\mu}^{a b}=\left(\delta_{\mu}^{b} \eta^{a v}-\delta_{\mu}^{a} \eta^{b v}\right) \partial_{\nu} \ln \rho
$$


Specifically in 2-D space-time with conformally flat co-ordinates $(\tau, \xi)$ :

$$
\omega_{\tau}^{01}=-\omega_{\tau}^{10}=-\partial_{\xi} \ln \rho, \quad \omega_{\xi}^{01}=-\omega_{\xi}^{10}=-\partial_{\tau} \ln \rho .
$$

Combining this result with (A9) and (A2), while noting that

$$
\sqrt{-g}=\operatorname{det} e_{\mu}^{a}=\rho^{2}
$$

one derives the result (35):

$$
\sqrt{-g} C \not D=\sqrt{\rho}\left(\begin{array}{cc}
\partial_{\tau}-\partial_{\tilde{\zeta}} & 0 \\
0 & \partial_{\tau}+\partial_{\xi}
\end{array}\right) \sqrt{\rho}
$$

But this is not yet the final result; starting form the Minkowski 2-bein $e_{M \mu}^{a}=\delta_{\mu}^{a}$, the Rindler 2-bein is obtained by

$$
e_{R \mu}^{a}=\frac{\partial x_{M}^{\lambda}}{\partial x_{R}^{\mu}} e_{M \lambda}^{b} \Omega_{b}^{a}
$$

where in the $R$-wedge of Rindler space

$$
\frac{\partial x_{M}^{\lambda}}{\partial x_{R}^{\mu}}=e^{a \xi}\left(\begin{array}{cc}
\cosh a \tau & \sinh a \tau \\
\sinh a \tau & \cosh a \tau
\end{array}\right)
$$

is the Jacobian of the transformation from Minkowski to Rindler co-ordinates, and $\Omega_{b}^{a}$ is a local Lorentz transformation. Clearly the Jacobian is not diagonal, but this can be restored by taking the local Lorentz transformation

$$
\Omega=\left(\begin{array}{cc}
\cosh a \tau & -\sinh a \tau \\
-\sinh a \tau & \cosh a \tau
\end{array}\right)
$$

This puts the 2-bein back to the diagonal form $e_{R \mu}^{a}=e^{a \xi} \delta_{\mu}^{a}$. For the $L$-wedge a similar procedure can be followed. As a result, the use of a diagonal vierbein in Rindler space must be accompanied by a corresponding local Lorentz transformation on spinor fields; in the present case this takes the form

$$
\lambda^{\prime}=e^{a \tau \gamma_{3} / 2} \lambda .
$$

This explains why in the $R$ - and $L$-wedges of Rindler space the redefinition (36) of the spinor fields by

$$
\begin{array}{ll}
R: & \psi_{ \pm}(\tau, \xi)=e^{ \pm a(\tau \pm \xi) / 2} \lambda_{ \pm}[t(\tau, \xi), x(\tau, \xi)], \\
L: & \psi_{ \pm}(\tau, \xi)=e^{\mp a(\tau \pm \xi) / 2} \lambda_{ \pm}[t(\tau, \xi), x(\tau, \xi)],
\end{array}
$$

reduces the Rindler Dirac-Majorana action formally to the Minkowski one.

\section{Appendix B. Thermal Correlations in Rindler Space}

The computation of the two-point correlation functions of the Rindler fields in the Minkowski vacuum start from Equations (49) and (53):

$$
\begin{array}{ll}
b_{R \kappa}|0\rangle_{b}=e^{-\pi \kappa} b_{L \kappa}^{*}|0\rangle_{b}, & b_{R \kappa}^{*}|0\rangle_{b}=e^{\pi \kappa} b_{L \kappa}|0\rangle_{b}, \\
\beta_{R \kappa}|0\rangle_{f}=-i e^{-\pi \kappa} \beta_{L \kappa}^{*}|0\rangle_{f}, & \beta_{R \kappa}^{*}|0\rangle_{f}=i e^{\pi \kappa} \beta_{L \kappa}|0\rangle_{f},
\end{array}
$$


and their conjugates:

$$
\begin{aligned}
& { }_{b}\langle 0| b_{R \kappa}^{*}=e^{-\pi \kappa_{b}}\langle 0| b_{L \kappa}, \quad{ }_{b}\langle 0| b_{R \kappa}=e^{\pi \kappa}{ }_{b}\langle 0| b_{L \kappa^{\prime}}^{*} \\
& { }_{f}\langle 0| \beta_{R \kappa}^{*}=i e^{-\pi \kappa}{ }_{f}\langle 0| \beta_{L \kappa}, \quad{ }_{f}\langle 0| \beta_{R \kappa}=-i e^{\pi \kappa}{ }_{f}\langle 0| \beta_{L \kappa}^{*} .
\end{aligned}
$$

The following chain of commutation relations and substitutions leads to the desired result:

$$
\begin{aligned}
{ }_{b}\left\langle 0\left|b_{R \kappa} b_{R \sigma}^{*}\right| 0\right\rangle_{b} & =2 \pi \delta(\kappa-\sigma)+{ }_{b}\left\langle 0\left|b_{R \sigma}^{*} b_{R \kappa}\right| 0\right\rangle_{b} \\
& =2 \pi \delta(\kappa-\sigma)+e^{-\pi(\kappa+\sigma)}{ }_{b}\left\langle 0\left|b_{L \sigma} b_{L \kappa}^{*}\right| 0\right\rangle_{b} \\
& =2 \pi \delta(\kappa-\sigma)\left(1+e^{-2 \pi \kappa}\right)+e^{-\pi(\kappa+\sigma)}{ }_{b}\left\langle 0\left|b_{L \kappa}^{*} b_{L \sigma}\right| 0\right\rangle_{b} \\
& =2 \pi \delta(\kappa-\sigma)\left(1+e^{-2 \pi \kappa}\right)+e^{-2 \pi(\kappa+\sigma)}{ }_{b}\left\langle 0\left|b_{R \kappa} b_{R \sigma}^{*}\right| 0\right\rangle_{b} .
\end{aligned}
$$

The solution of this equation is (55) and implies (56):

$$
\begin{aligned}
& { }_{b}\left\langle 0\left|b_{R \kappa} b_{R \sigma}^{*}\right| 0\right\rangle_{b}=2 \pi \delta(\kappa-\sigma)+{ }_{b}\left\langle 0\left|b_{R \sigma}^{*} b_{R \kappa}\right| 0\right\rangle_{b}=\frac{2 \pi \delta(\kappa-\sigma)}{1-e^{-2 \pi \kappa}}, \\
& { }_{b}\left\langle 0\left|b_{R \sigma}^{*} b_{R \kappa}\right| 0\right\rangle_{b}=\frac{2 \pi \delta(\kappa-\sigma)}{e^{2 \pi \kappa}-1} .
\end{aligned}
$$

The derivation of the fermion two-point function proceeds entirely parallel, using anti-commutation relations instead of commutation relations, which results in a change of sign in the denominator.

The relations (A15), (A16) also imply a direct relation between the common Rindler vacuum state $|0\rangle_{R}=|0\rangle_{R R} \otimes|0\rangle_{R L}:$

$$
b_{R \kappa}|0\rangle_{R R}=\beta_{R \kappa}|0\rangle_{R R}=0, \quad b_{L \mathcal{K}}|0\rangle_{R L}=\beta_{L \kappa}|0\rangle_{R L}=0,
$$

and the Minkowski vacuum:

$$
|0\rangle_{M}=\exp \left[\int_{0}^{\infty} \frac{d \kappa}{2 \pi} e^{-\pi \kappa}\left(b_{R \kappa}^{*} b_{L \kappa}^{*}-i \beta_{R \kappa}^{*} \beta_{L \kappa}^{*}\right)\right]|0\rangle_{R} .
$$

\section{References}

1. Belavin, A.A.; Polyakov, A.M.; Zamolodchikov, A.B. Infinite conformal symmetry in two-dimensional quantum field theory. Nucl. Phys. B 1984, 241, 333-380.

2. Cardy, J.L. Conformal Invariance and Statistical Mechanics. In Les Houches Summerschool in Theoretical Physcis (1988); Brezin, E., Zinn-Justin, J., Eds.; 1990. Available online: https://inspirehep.net/literature/25027 (accessed on 3 September 2020).

3. Schellekens, A.N. Conformal Field Theory; Lectures at the Saalburg Summerschool 1995. Available online: https:/ / www.nikhef.nl/ t58/CFTBW.pdf (accessed on 3 September 2020).

4. Green, M.B.; Schwarz, J.H.; Witten, E. Superstring Theory; Cambridge University Press: Cambridge, UK, 1987.

5. Fradkin, E. Field Theories of Condensed Matter Physics; (Cambridge University Press: Cambridge, UK, 2013.

6. Zaanen, J.; Sun, Y-.; Liu, Y.; Schalm, K. Holographic Duality in Condensed Matter Physics; Cambridge University Press: Cambridge, UK, 2015.

7. Rindler, W. Kruskal Space and the Uniformly Accelerated Frame.Am. J. Phys. 1966, 34, 1174.

8. Unruh, W.G. Notes on black-hole evaporation. Phys. Rev. D 1976, 14, 870.

9. Davies, P.C.W. Scalar production in Schwarzschild and Rindler metrics. J. Phys. A 1975, 8, 609.

10. Wald, R.M. On particle creation by black holes. Comm. Math. Phys. 1975, 45, 9-34.

11. Fulling, S.A. Alternative vacuum states in static space-times with horizons. J. Phys. A 1977, 10, 917. 
12. Hawking, S.W. Black hole explosions? Nature 1974, 248, 30-31.

13. Hawking, S.W. Particle creation by black holes. Comm. Math. Phys. 1975, 43, 199-200.

14. Gibbons, G.W.; Hawking, S.W. Cosmological event horizons, thermodynamics, and particle creation. Phys. Rev. D 1977, 15, 2738.

15. Samantray, P.; Padmanabhan, T. Conformal symmetry, Rindler space, and the AdS/CFT correspondence. Phys. Rev. D 2014, 90, 47502.

16. Brout, R.; Massar, S.; Parentani, R.; Spindel, P. A Primer for Black Hole Quantum Physics. Phys. Rept. $1995,260,329$.

17. Crispino, L.C.; Higuchi, A.; Matsas, G. The Unruh effect and its applications. Rev. Mod. Phys. 2008, 80, 787.

18. Wald, R.M. General Relativity; University of Chicago Press: Chicago, IL, USA, 1984.

19. Fulling, S.A. Aspects of Quantum Field Theory in Curved Space-Time; Cambridge University Press: Cambridge, UK, 1989

20. Siegel, W. Covariantly second-quantized string. Phys. Lett. B 1984, 142, 276.

21. Petcher, D.N.; van Holten, J.W. A note on Fujikawa's method of determining the critical dimension of the relativistic string. Phys. Lett. B 1987, 194, 221.

22. van Holten, J.W.; Kowalski-Glikman, J.; Petcher, D.N. BRST invariance and gauge independence of string path-integrals. Nucl. Phys. B 1988, 309, 608.

23. Wess, J.; Zumino, B. Consequences of anomalous ward identities. Phys. Lett. B 1971, 37, 95.

(C) 2020 by the author. Licensee MDPI, Basel, Switzerland. This article is an open access article distributed under the terms and conditions of the Creative Commons Attribution (CC BY) license (http://creativecommons.org/licenses/by/4.0/). 